International Journal of Cancer Studies \& Research (IJCR)

ISSN: $2167-9118$

\title{
Stochastic Mathematical Models of Carcinogenesis
}

Tan WY

Editorial

Department of Mathematical Sicences, University of Memphis, Memphis, TN, USA.

\section{*Corresponding Author:}

Wai-Yuan Tan,

Department of Mathematical Sicences, University of Memphis, Memphis, TN, USA.

E-mail: waitan@memphis.edu

Accepted: August 16, 2012

Published: October 02, 2012

Citation: Tan WY (2012) Stochastic Mathematical Models Of Carcinogenesis. Int J Cancer Stud Res. 1(1e), 1. doi: http://dx.doi. org/10.19070/2167-9118-120001e

Copyright: Tan WY $\mathbf{Y}^{\mathcal{O}}$ 2012. This is an open-access article distributed under the terms of the Creative Commons Attribution License, which permits unrestricted use, distribution and reproduction in any medium, provided the original author and source are credited.

Geneticists and molecular biologists have demonstrated that carcinogenesis is a stochastic multi-stage process with each cancer tumor being derived from a single stem cell which has sustained a finite number of genetic and/or epigenetic changes (Tan 1991, Wienberg 2007, Tan, Chen and Zhang, 2008). These genetic and/ or epigenetic changes initiate and facilitate cancer progression by driving and disturbing some relevant molecular signal pathways to promote cell proliferation and growth of cancer stem cells. In order for the biological findings to be tested quantitatively against human epidemiological data and animal experimental data, and in order to develop efficient controlling strategies for human cancer, it is absolutely essential that these biological findings be transformed into stochastic mathematical models. This calls for the development of biologically based stochastic mathematical models for human cancers.

To serve as an example, consider the human colon cancer which has been shown to be developed mainly by two multi-stage pathways- the APC--Catenin-Tcf-myc pathway (denoted by N -> I1 -> I2 -> I3 -> I4 -> Tumor, a 4-stage stochastic pathway) and the Micro- Satellite Instability pathway (denoted by N -> J1 -> J2 -> J3 -> J4 -> J5 -> Tumor, a 5-stage stochastic pathway) (Tan et al. 2008). Biologists have shown that the I2 cells and the J3 cells divide stochastically to generate polyps which form the basis for the colon screening procedure "colonoscopy". To evaluate the efficiency and usefulness of this cancer screening rocedure it is absolutely necessary to develop stochastic models for human colon cancer. To serve as another example, consider the pediatric human eye cancer-retinoblastoma which is initiated by the mutation or deletion or inactivation of the retinoblastoma gene (the Rb gene) in chromosome 13q. As many other pediatric human cancers (e.g. Wilms's tumor, hepatoblastoma, medulloblastoma, etc.), for retinoblastoma a large number of cancer cases develop at and before birth during pregnant period. This calls for the development of stochastic models involving population genetics to involve inherited cancer cases.

Based on the above and many other molecular and genetic findings (Weinberg, 2007), this short note calls for the development of stochastic mathematical models for human pediatric and adult cancers. To conclude, we quote Weinberg (2007, Page 794. The Biology of Cancer. Garland Sciences, Taylor and Frances, New York): "Image a day-still years away-when the biological responses of various human cells, normal and malignant, can be predicted by mathematical models of these cells and their internal circuits. Such advances will render many current practices in experimental biology, including many steps of drug development, unnecessary. If this even becomes possible, drug development will be more a matter of dry bioinformatics than wet biology at the laboratory bench."

\section{References}

[1]. Tan, W.Y.: Stochastic Models of Carcinogenesis. Marcel Dekker, New York, 1991.

[2]. Weinberg, R.A.: The Biology of Human Cancer. Garland Sciences, Taylor and Frances Group, New York, 2007.

[3]. Tan, W.Y., Chen, C.W. and Zhang,L.J.: Cancer Biology, Cancer models and Stochastic Mathematical Analysis of Carcinogenesis. In: " Handbook of Cancer Models and Applications." eds. Tan,W.Y. and Hanin, L. Chapter 3, World Scientific, River Edge, NJ., 2008.

[4]. Tan W.Y., Zhang L.J., Chen W. and Zhu J.M.: A stochastic model of human colon cancer involving multiple pathways. In: "Handbook of Cancer Models with Applications." eds. Tan W.Y. and Hanin L.Chapter 11, World Scientific, River Edge, NJ., 2008. 